\title{
A Single Dose of the Selective Serotonin Reuptake Inhibitor Citalopram Exacerbates Anxiety in Humans: A Fear-Potentiated Startle Study
}

\author{
Christian Grillon*,', Jessica Levenson' and Daniel S Pine' \\ 'Mood and Anxiety Disorders Program, National Institute of Mental Health, NIH, Bethesda, MD, USA
}

\begin{abstract}
Serotonin reuptake inhibitors may increase symptoms of anxiety immediately following treatment initiation. The present study examined whether acute citalopram increased fear-potentiated startle to predictable and/or unpredictable shocks in healthy subjects. Eighteen healthy subjects each received two treatments, placebo and $20 \mathrm{mg}$ citalopram in a crossover design. Participants were exposed to three conditions including one in which predictable aversive shocks were signaled by a cue, a second in which unpredictable shocks were anticipated, and a third in which no shocks were administered. Changes in aversive states were investigated using acoustic startle stimuli. Citalopram did not affect baseline startle. However, the phasic startle potentiation to the threat cue in the predictable condition was robustly increased by acute citalopram. The sustained startle potentiation in the unpredictable conditions was also increased by citalopram, but only when the drug was given during the first session. These results indicate that a single dose of citalopram is not anxiogenic in itself, but can exacerbate the expression of fear and anxiety.

Neuropsychopharmacology (2007) 32, 225-23I. doi:I0.I038/sj.npp. I30I204; published online I3 September 2006
\end{abstract}

Keywords: fear; anxiety; SSRI; citalopram; startle reflex; predictability

\section{INTRODUCTION}

Serotonin reuptake inhibitors (SSRIs) have become the medication of choice for anxiety and depression, partly because they cause fewer side effects compared to other psychopharmacological treatments. Several studies report symptom improvement in various anxious patient populations following chronic SSRIs. However, the influence of these drugs is not uniform over time. Shortly after treatment initiation, a substantial number of patients report exacerbation characterized by 'jitteriness', anxiety, nervousness, restlessness, agitation, irritability, accompanied by sleep and gastrointestinal disturbance (Gorman et al, 1987; Ramos et al, 1993). This exacerbation of symptoms can be an obstacle to treatment continuation; dropout rates as high as $50 \%$ have been reported (Gorman et al, 1987).

The mechanisms involved in the anxiolytic and anxiogenic effects of SSRIs are currently unclear. The initially anxiogenic-like effects may result from an acute increased availability of serotonin on inhibitory $5 \mathrm{HT}_{1 \mathrm{~A}}$ autoreceptors,

\footnotetext{
*Correspondence: Dr C Grillon, Mood and Anxiety Disorders Program, National Institute of Mental Health, NIH, I5K North Drive, Bldg I5K, Room II3, MSC 2670, Bethesda, MD 20892-2670, USA, Tel: + I 30| 594 2894, Fax: + | 30 I 594 9959,

E-mail: Christian.grillon@nih.gov

Received 22 May 2006; revised 13 July 2006; accepted 26 July 2006 Online publication: 4 August 2006 at http://www.acnp.org/citations/ Npp080406060336/default.pdf
}

leading to a reduced firing of ascending serotonin raphe neurons, until adaptive changes ensue following chronic treatment (Piñeyro and Blier, 1999). Alternatively, serotonin could affect multiple mechanisms of anxiety. In animals, acute serotonin can increase (Bagdy et al, 2001; Griebel et al, 1994) or decrease (Inoue et al, 1996, 2004; Sanchez and Meier, 1997) anxiety-like responses, depending on the model used. One potential interpretation of these findings is that different animal models represent qualitatively different types of fear or anxiety. This would be consistent with the hypothesis that serotonin has effects on multiple brain structures that mediate anxiety via multiple pathways and receptors (Graeff et al, 1997).

Little is known about the effect of SSRIs on experimental anxiety in humans. Acute citalopram facilitates the cortical processing of pleasant stimuli, while reducing the processing of unpleasant stimuli, thus shifting attentional bias from negative to positive stimuli (Kemp et al, 2004). Acute citalopram enhances the processing of facial expression of fear and happiness, but not sadness, anger, and disgust, suggesting a selective effect (Harmer et al, 2003b). However, the manner in which SSRIs affect emotional responses to more evocative threat cues remains unclear.

Increased understanding of the effect of SSRIs on aversive states may elucidate the psychopharmacological bases of aversive behaviors. As an initial step towards this aim, we investigated the effects of a single dose of citalopram on fear-potentiated startle in humans. A verbal threat proce- 
dure was implemented instead of a conditioning procedure given that abnormal responses to verbal threat more consistently characterize clinical anxiety states, relative to conditioning procedure (Lissek et al, 2005a,b). Moreover, this design focuses on modulatory influences on expressions of aversive responses rather than learning and memory processes inherently associated with associative learning procedures (Grillon and Baas, 2003).

Following up on works by Davis and collaborators using the startle reflex (Walker et al, 2003), we identified two threat responses: a phasic fear-potentiated startle response associated with a cue and a more sustained startle potentiation generated by less explicit threat (Grillon et al, 2005,2004 ) by administering predictable (ie signaled by a cue) or unpredictable (ie unsignaled) shocks, respectively. The main objective of this study was to examine the effect of citalopram on these two types of responses.

It was expected that acute citalopram would facilitate phasic cued fear-potentiated startle. This hypothesis was based on converging evidence that the processing of and reaction to threat cues is modulated by SSRIs. Thus, acute citalopram enhances cued fear conditioning in rodents (Burghardt et al, 2004) and facilitates the processing of fear faces in humans (Harmer et al, 2003b). In addition, acute administration of serotonin-receptor blockers resanterin decreases conditioned responses to aversive loud noises (Hensman et al, 1991; Silva et al, 2001). No hypothesis was formulated concerning the effect of acute citalopram on sustained potentiated startle to unpredictable shocks because the pre-clinical literature has provided conflicting evidence. For example, SSRIs have different effects on two models of sustained anxiety, contextual fear, and lightenhanced startle. Citalopram impairs context conditioning (Inoue et al, 1996, 2004) but fluvoxamine does not affect light-enhanced startle (de Jong et al, 2002).

\section{METHODS}

\section{Participants}

Participants were healthy volunteers who gave written informed consent approved by the NIMH Human Investigation Review Board. Inclusion criteria included (1) no past or current psychiatric disorders as per Structured Clinical Interview for DSM-IV (SCID: (First et al, 1995)), (2) no medical condition that interfered with the objectives of the study as established by a physician, and (3) no use of elicit drugs or psychoactive medications as per urine screen.

Participants underwent a screening session that consisted of a SCID, a physical exam, and a shock workup procedure to establish a level of shock that was 'highly annoying but not painful.' In addition, subjects were screened for baseline startle reactivity with nine startle stimuli (40-ms duration, $103 \mathrm{~dB}$ ). Subjects who displayed small startle responses (a mean of less than $5 \mu \mathrm{V}$ over nine startle responses), or no startle response on at least one trial were not invited to participate in the study. Four to 10 days after screening, participants returned for the first of two testing sessions. Eighteen subjects (nine males) with a mean age of 26.5 years $(S D=7.9$ years) were ultimately included in the study with a mean trait anxiety score based on Spielberger's
State and Trait Anxiety Inventory (Spielberger, 1983) of 25.8 $(\mathrm{SD}=3.8)$.

\section{Drug Manipulation}

The treatments were $20 \mathrm{mg}$ citalopram and placebo, tested in a double-blind crossover design (within-subjects). The order of treatment was counterbalanced across subjects. Half the subjects were given placebo during the first session.

\section{Procedure}

The procedure was similar to that of a recent study that tested the effect of alprazolam (Grillon et al, in press). On the test day, the electrodes to measure the eyeblink were applied. Next, subjects ingested a capsule containing citalopram or placebo before resting for $2 \mathrm{~h}$ to allow drug absorption, after which the shock electrodes were attached.

To test the effect of citalopram on startle reactivity, two blocks of nine startle stimuli (inter-startle stimulus interval: 18-25s) were presented. One block was given just before ingestion of the drug (pre-drug startle) and the other $2 \mathrm{~h}$ later (post-drug startle), just before application of the shock electrodes. Participants were then given explicit instructions regarding the conditions under which they would and would not receive a shock. Following the instructions, the threat experiment began. The experiment consisted of three different conditions, a no shock condition $(\mathrm{N})$, and two conditions during which shocks were administered either predictably $(\mathrm{P})$, that is, only in the presence of a threat cue, or unpredictably (U). Each condition lasted approximately $150 \mathrm{~s}$. In each 150-s condition, an 8-s cue was presented four times. The cues were different geometric colored shapes in the different conditions (eg blue square for $\mathrm{N}$, red circle for $\mathrm{P})$. The cues signaled the possibility of receiving an aversive stimulus only in the $\mathrm{P}$ condition, but had no signal value in the $\mathrm{N}$ and $\mathrm{U}$ conditions. Participants were verbally instructed regarding the risk of shock in the different conditions and they were informed of the contingency or lack of contingency between shock and cues in the $\mathrm{P}$ and $\mathrm{U}$ conditions. In addition, instructions were also shown on a computer monitor throughout the experiment displaying the following information: 'no shock' (N), 'shock only during shape' $(\mathrm{P})$, or 'shock at any time' (U). During each predictable and unpredictable condition, one shock was administered, during the cue in the predictable condition and in the absence of the cues in the unpredictable condition. In each $\mathrm{N}, \mathrm{P}$, and $\mathrm{U}$ condition, six acoustic startle stimuli were delivered, three during inter-trial intervals (ITI; that is, between cues) and one during three of the four cues, 5-7 s following cue onset. The threat experiment consisted of two recording blocks with a 5-10 min rest between blocks. Each block started with the delivery of six startle stimuli (pre-test startle) and consisted of three $\mathrm{N}$, two $\mathrm{P}$, and two $\mathrm{U}$ conditions in one of the following two orders: P N U N U N P or U N P N P N U. Each participant was presented with the two orders, with half the participants starting with the $\mathrm{P}$ condition. One shock was administered in each individual $\mathrm{P}$ and $\mathrm{U}$ condition for a total of four shocks in the four $\mathrm{P}$ conditions and four shocks in the four $U$ conditions. The shock was delivered $7.5 \mathrm{~s}$ following cue onset in the P condition. It was 
administered either 7 or $10 \mathrm{~s}$ following cue offset in the unpredictable condition. No startle stimuli could follow a shock by less than $10 \mathrm{~s}$.

Questionnaires were given just before taking the treatments and $2 \mathrm{~h}$ later to assess the effects of the treatment. They included Spielberger's state portion of the state-trait anxiety inventory questionnaire (Spielberger, 1983), and a mood rating scale that evaluated subjective feelings of mental and physical sedation. In addition, after each recording block, subjects retrospectively rated how anxious they felt in the presence and absence of the cue in each condition $(\mathrm{N}, \mathrm{P}, \mathrm{U}$ ) on an analog scale ranging from 0 (not at all anxious) to 10 (extremely anxious).

\section{Stimuli and Physiological Responses}

Stimulation and recording were controlled by a commercial system (Contact Precision Instruments, London, UK). The acoustic startle stimulus was a $40-\mathrm{ms}$ duration, $103 \mathrm{~dB}$ (A) burst of white noise with a near instantaneous rise time presented binaurally through headphones. The eyeblink reflex was recorded with electrodes placed under the left eye. Amplifier bandwidth was set to $30-500 \mathrm{~Hz}$. The electric shock was produced by a constant current stimulator and administered on the left wrist.

\section{Data Analysis}

Peak amplitude of the blink reflex was determined in the $20-100$-ms time frame following stimulus onset relative to baseline (average baseline EMG level for the $50 \mathrm{~ms}$ immediately preceding stimulus onset) and averaged within each condition. The startle data and retrospective measures of subjective anxiety were analyzed with analyses of variance (ANOVA) with repeated measures. Preliminary analyses indicated no sex difference for the startle and subjective measures. Hence, sex was not entered as a factor in the data analysis. Alpha was set at 0.05 for all statistical tests. Greenhouse-Geisser corrections (GG- $\varepsilon$ ) were used for main effects and interactions involving factors with more than two levels.

\section{RESULTS}

\section{Startle Data}

Effect of citalopram. The pre-drug and post-drug baseline startle magnitudes are shown in Table 1. Baseline startle was not affected differently by placebo and citalopram. A Treatment (placebo, citalopram) $\times$ Time (pre-drug, post drug) $\times$ Order (placebo first session, citalopram first session) ANOVA revealed no significant main effect or interaction effect (all $p>0.1$ ) on baseline startle.

Early contextual effect. Placing the shock electrodes and starting a threat experiment can potentiate startle (Grillon and Ameli, 1998). To examine the effect of citalopram on this type of startle potentiation, post-drug startle magnitude was compared to the pre-test startle (ie in responses to the leading startle stimuli delivered at the beginning of the first threat block). Table 1 shows that the increase in startle magnitude from post-drug to pre-test was greater with citalopram compared to placebo. Statistical analyses using a Treatment (placebo, citalopram) $\times$ Time (post-drug, pre-test) $\times$ Order (placebo first session, citalopram first session) ANOVA confirmed this finding. There was a significant Time main effect $(\mathrm{F}(1,16)=9.8, p<0.006)$ that was qualified by a significant Time $\times$ Treatment interaction $(\mathrm{F}(1,16)=6.1, p<0.02)$, with no significant Treatment $\times$ Order or Time $\times$ Treatment $\times$ Order effect $(p>0.1)$. Follow-up $t$-tests revealed that the increased startle magnitude was significant with citalopram $(+14.5 \mu \mathrm{V}$, SEM $=3.6 \mu \mathrm{V}: \mathrm{t}(17)=3.9, p<0.001)$ but not with placebo $(+3.4 \mu \mathrm{V}, \mathrm{SEM}=3.6 \mu \mathrm{V}: \mathrm{t}(17)=0.9, \mathrm{NS})$.

Effect of citalopram of fear-potentiated startle to the threat cue. Startle magnitudes in each condition and in each treatment are presented in Table 1. As expected (Grillon et al, 2004), the increase in startle during the cues relative to ITI (fear-potentiated startle) was larger in the predictable condition compared to the two other conditions (Condition $\times$ Cue; $F(2,32)=21.2, p=0.001)$. Startle was potentiated by the cue relative to ITI in the predictable condition $(\mathrm{F}(1,17)=31.1, p<0.0009)$ and to a lesser extent in the unpredictable condition $(\mathrm{F}(1,17)=8.4, p<0.01)$, but not in the no shock condition. Importantly, the magnitude of fearpotentiated startle to the cues was affected differently by the two treatments (Treatment $\times$ Condition $\times$ Cue interaction: $\mathrm{F}(2,32)=3.2, p<0.05, \mathrm{GG}-\varepsilon=0.97$ ). (There was no significant effect with the factor Order.) To facilitate the analysis of the three-way interaction, fear-potentiated startle scores were computed by calculating the difference scores between startle magnitude during the cues and startle magnitude during ITI (Figure 1). Planned comparisons revealed significantly greater fear-potentiated startle effects with citalopram compared to placebo in the predictable condition $(\mathrm{F}(1,17)=4.7, \quad p<0.04)$. No differential effect of treatment was found in the unpredictable condition $(\mathrm{F}(1,17)=0.1, \mathrm{NS})$.

Table I Mean (SEM) Startle Magnitude $(\mu \mathrm{V})$ before and after Treatment and during the Cue and ITI Across Treatments and Conditions

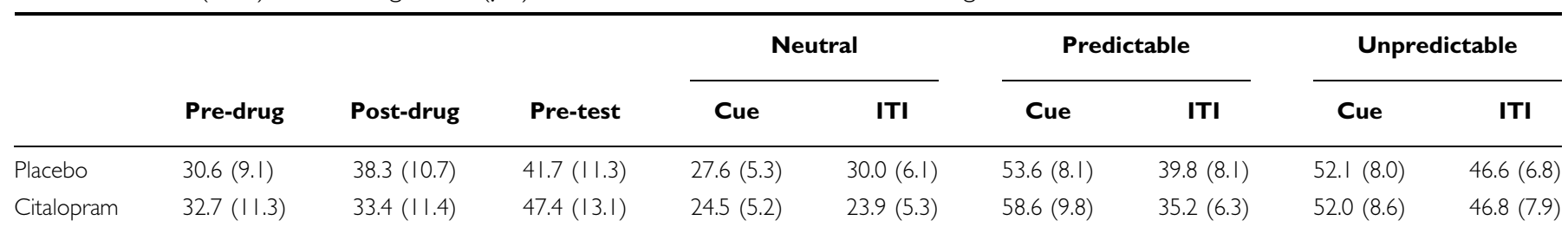

ITI, inter-trial interval; SEM, standard error of mean. 


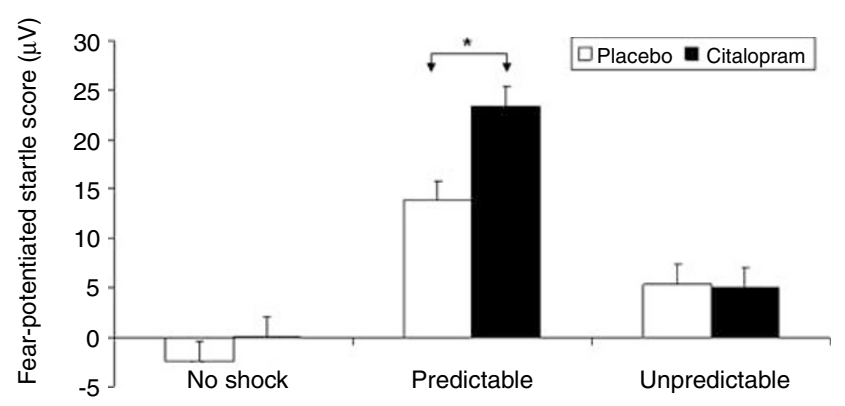

Figure I Phasic fear-potentiated startle response in each treatment: Difference scores reflecting cue minus ITI startle magnitudes in the no shock, predictable, and unpredictable conditions. *Significant $(p<0.05)$ difference between placebo and citalopram.

Contextual anxiety. Contextual anxiety was evaluated using the ITI startle data (Table 1), which were entered into a Treatment (placebo, citalopram) $\times$ Condition $(\mathrm{N}, \mathrm{P}$, $\mathrm{U}) \times$ Order (placebo first session, citalopram first session) ANOVA. This analysis contrasts with the earlier context analysis by focusing on ITI startle responses throughout the entire experiment. There was a Condition main effect $(\mathrm{F}(2,32)=16.8, p<0.0009, \mathrm{GG}-\varepsilon=0.83)$, owing to a progressive increase in ITI startle amplitude from the no shock, to the predictable, to the unpredictable condition (Linear trend: $(\mathrm{F}(1,16)=19.6, p<0.0009)$, a trend for a Treatment $\times$ Condition $\quad(\mathrm{F}(2,32)=2.7, \quad p<0.08, \quad \mathrm{GG}-\varepsilon=0.83)$ interaction, and a significant Treatment $\times$ Condition $\times$ Order interaction $(\mathrm{F}(2,32)=3.9, p<0.03, \mathrm{GG}-\varepsilon=0.83)$. To better understand the nature of the three-way interaction, we calculated the increase in ITI startle magnitude from the neutral condition to the predictable and unpredictable conditions (predictable minus neutral and unpredictable minus neutral). The resulting scores were entered into separate Treatment (placebo, citalopram $) \times$ Condition $(\mathrm{P}$, $\mathrm{U})$ session ANOVAs for subjects who were given placebo in the first session (and citalopram in the second session) and for subjects who were given citalopram in the first session (and placebo in the second session). Results showed that startle was significantly increased by citalopram when subjects received citalopram during the first session $(\mathrm{F}(2,16)=3.9, \quad p<0.04, \mathrm{GG}-\varepsilon=0.85)$, not when subjects were given placebo in the first session $(\mathrm{F}(2,16)=0.6$, NS). These effects are shown in Figure 2.

\section{Mood Ratings}

The mental and physical rating scores of one subject were lost. Subjects showed no significant effect of citalopram on state anxiety, and on mental and physical sedation (Table 2). Data were analyzed using separate Treatment (placebo, citalopram) $\times$ Time (pre-drug, post drug) $\times$ Order (placebo first session, citalopram first session) ANOVAs. For state anxiety and mental sedation, there were significant Time effect, due to a slight increase in state anxiety $(\mathrm{F}(1,16)=5.5$, $p<0.03)$, and mental $(\mathrm{F}(1,15)=4.8, p<0.04)$ and physical $(\mathrm{F}(1,15)=13.6, p<0.0009)$ sedation after both treatments. However, there was no significant differential effect of treatment on these ratings (no significant Condition $x$ Treatment).

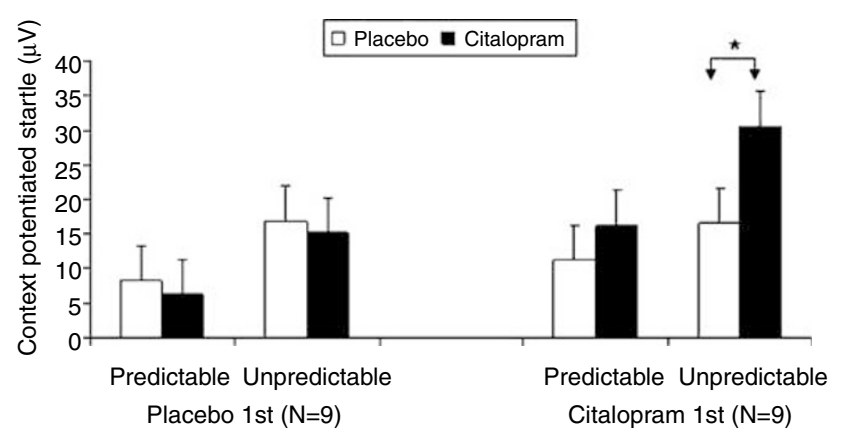

Figure 2 Sustained potentiated startle responses in each treatment: Difference scores between ITI startle magnitudes in the threat conditions (predictable and unpredictable) and ITI startle magnitude in the no shock condition. *Significant $(p<0.05)$ difference between placebo and citalopram.

Table 2 Mean (SEM) State Anxiety, and Physical and Mental Sedation before and after Treatment

\begin{tabular}{lcclll}
\hline & \multicolumn{2}{c}{ Physical sedation } & & \multicolumn{2}{c}{ Mental sedation } \\
\cline { 2 - 3 } \cline { 6 - 6 } & Pre-drug & Post-drug & & Pre-drug & Post-drug \\
\hline Placebo & $9.5(0.9)$ & $11.8(1.2)$ & & $11.0(1.3)$ & $12.1(1.4)$ \\
Citalopram & $10.8(1.3)$ & $13.1(1.5)$ & & $12.4(1.5)$ & $13.6(1.7)$ \\
\hline
\end{tabular}

SEM, standard error of mean.

\section{Retrospective Ratings of Anxiety}

The subjective anxiety ratings were analyzed in a similar way as the eyeblink data.

Cued fear. The retrospective anxiety rating scores are shown in Table 3. Subjective anxiety to the cues was differentially affected by the contexts (Condition $\times$ Stimulus Type; $\mathrm{F}(2,30)=17.2, p<0.0009, \mathrm{GG}-\varepsilon=0.91)$. Subjective anxiety was higher during the cue compared to ITI in the predictable condition $(\mathrm{F}(1,17)=17.3, p<0.001)$, but not in the unpredictable $(\mathrm{F}(1,17)=2.8$, NS) or the no shock $(\mathrm{F}(1,17)=0.1, \mathrm{NS})$ conditions. The increased anxiety in the predictable condition was not affected by the treatment $(\mathrm{F}(1,17)=0.9, \mathrm{NS})$.

Contextual anxiety. Like ITI startle amplitude, subjective anxiety increased linearly from the no shock, to the predictable, and to the unpredictable condition $(\mathrm{F}(1,16)=$ $146.3, p<0.0009$ ). Relative to placebo, citalopram did not significantly affect subjective anxiety, as reflected by a lack of Treatment main effect $(F(1,16)=0.7, N S)$ or Condition $\times$ Treatment interaction $(\mathrm{F}(2,32)=2.5$, NS).

\section{DISCUSSION}

The present study found that startle amplitude was not significantly affected by citalopram before the threat test was initiated. In contrast, citalopram increased the magnitude of multiple forms of startle potentiation, including fear potentiated to a discrete threat cue, sustained potentiated 
Table 3 Mean (SEM) Retrospective Rating of Anxiety during the Cue and ITI Across Treatments and Conditions

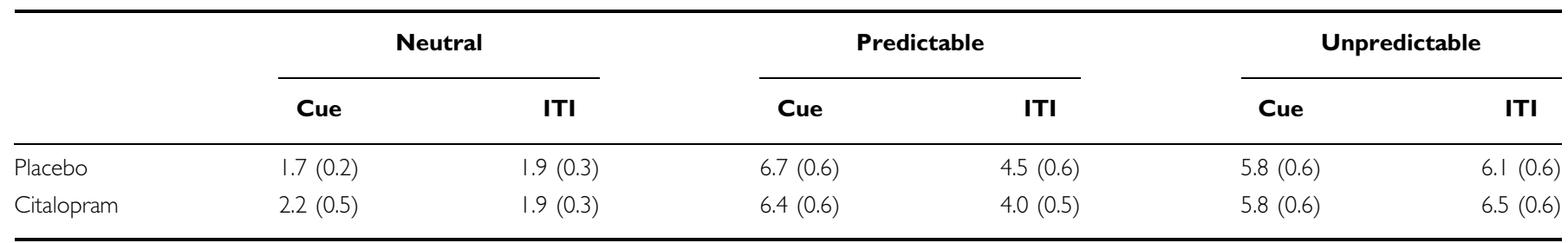

ITI, inter-trial interval; SEM, standard error of mean.

startle to unpredictable shock, and the contextual startle potentiation in the early phase of the threat experiment. These results suggest that citalopram was not anxiogenic per se in the absence of danger, but that it can exacerbate anxiety experienced in responses to threat. This later effect is consistent with clinical evidence of anxiogenic reactions, shortly following treatment initiation with SSRIs in patients with anxiety disorders.

In rodents, acute citalopram increases the acquisition of cued fear conditioning (Burghardt et al, 2004). This effect was attributed (Burghardt et al, 2004) to better learning, based on the finding that acute citalopram facilitates recall and recognition of verbal material in humans (Harmer et al, 2002). Acute citalopram also facilitates the recognition of fear from facial expression, suggesting enhancement of the processing of threatening cue in the environment independent of any effect on memory acquisition (Harmer et al, $2003 \mathrm{~b})$. It is unlikely that the facilitation of cued fearpotentiated startle in the present study was due to either better learning/memory process or enhanced recognition of threat cues. Subjects were instructed of the contingency between the shock and the threat cue, and instructions concerning the various threat conditions were clearly displayed on the monitor facing the subjects throughout testing (See Methods). Prior studies with this paradigm show that threat cues are very easy to discriminate from safe cues (Grillon et al, 1991). The findings also cannot be due to enhanced recognition of the threat signal because fear was probed with the startle reflex several seconds after cue onset, leaving subjects ample time to recognize the non-ambiguous threat cue. The most straightforward interpretation of the results is that acute citalopram facilitated the expression of an aversive response. Taken together, these results indicate that acute SSRIs can affect multiple levels (eg perception, expression) of the aversive motivational system(s). Hence, acute SSRIs may act to enhance fear/anxiety independent of its facilitative effects on more intermediary encoding and retrieval processing stages. All these effects may be causally related to the multitude of symptoms experienced by patients following treatment initiation with SSRIs.

The subjective data did not match the startle findings. Similar dissociations between objective measures and subjective reports have been reported. The increased recognition of fearful faces with a single dose of citalopram arises in the absence of subjective mood changes (Harmer et al, 2003b). Similarly, acute citalopram affects behavioral, cortical, and physiological responses to emotional stimuli in the absence of conscious changes in subjective feelings (Kemp et al, 2004). One obvious reason for the differential effect of citalopram on physiological and subjective reports in the present study is that startle was used to probe anxiety online, whereas the subjective anxiety measures were retrospective. The passage of time may have obscured subtle differences in responding because of the complexity of the design. Alternatively, startle and subjective ratings may reflect distinct processes differently sensitive to acute citalopram. Startle is a low-level response and verbal report of emotions involves more elaborate cognitive activity. Potentially, startle may be able to uncover early drug effects before changes in subjective feelings. This would be consistent with findings that the amygdala can be activated without alteration in conscious mood (Morris et al, 1998).

Phasic responses to a predictable threat cue and sustained responses to unpredictable shocks were investigated because of evidence that these responses are activated by distinct neural mechanisms in rodents (Walker et al, 2003). More specifically, fear-potentiated startle to a discrete cue is mediated by the amygdala, whereas the more sustained startle potentiation is mediated by both the amygdala and the bed nucleus of the stria terminalis (BNST) (Walker et al, 2003). We recently reported a psychopharmacological dissociation of responses of these two types of responses in humans. The benzodiazepine alprazolam reduced potentiated startle to unpredictable shocks, but not to predictable shocks (Grillon et al, in press). In the present study, there was no strong differential effect of citalopram on responses to predictable and unpredictable shocks. Although acute citalopram led to a robust increase in cued fear independent of treatment order, it facilitated the sustained startle potentiation in the unpredictable condition only in subjects who received citalopram during the first session (and placebo in the second session). In addition, citalopram increased baseline startle during the early phase of the threat experiment (when subjects were not at imminent risk of receiving a shock), reflecting an initial potentiation of contextual fear. This effect did not last as suggested by the fact that there was no effect of treatment on ITI startle in the no shock condition. One common factor between these two aversive situations (unpredictable condition and early contextual anxiety) is that the intensity of anxiety elicited diminished with time. Anxiety to unpredictable shocks is usually the strongest during the first of multiple sessions (unpublished observation) and contextual anxiety is stronger earlier compared to later in the experiment. Hence, one potential interpretation for the selective effect of citalopram is that this drug exacerbated anxiety responses only when these responses were sufficiently intense, but not when they were weaker. This explanation should be tested by examining the effects of citalopram on different levels of fear.

Serotonin modulation in the amygdala alone or in combination with serotonin modulation in the BNST 
following a single dose of citalopram could explain startle potentiation to the different types of threat. The amygdala, which receives serotoninergic innervations from the median raphe nucleus (Vertes et al, 1999), is involved in fear to verbal threat (Phelps et al, 1998; Funayama et al, 2001). Consistent with this hypothesis, the amygdala is also involved in other types of responses that are facilitated by acute citalopram, that is, cued fear conditioning (Burghardt et $a l, 2004$ ) and the processing of fearful faces (Harmer et al, 2003a). The amygdala is also involved in the initiation of sustained aversive responses (Walker et al, 2003). Furthermore, acute treatment with SSRIs increases c-fos immunoreactivity in the amygdala (Lino-de-Oliveira et al, 2001). It is also possible that serotonin modulation in the BNST mediates the anxiogenic effects of citalopram on responses to unpredictable shocks given that this structure, which modulates response to unpredictable stressors, also receives serotoninergic innervations from the raphe nucleus (Phelix et al, 1992). However, at the present time, little is known about the role of the BNST in humans.

The anxiogenic effects of acute citalopram are likely to be mediated by action on the reuptake of serotonin because citalopram has minimal effect on dopamine and noradrenalin transporters and has little affinity for a variety of neurotransmitter receptors (Noble and Benfield, 1997; Pollock, 2001). An acute reduction in serotonin availability secondary to activation of $5 \mathrm{HT}_{1 \mathrm{~A}}$ autoreceptors has been proposed as a potential mechanism (Piñeyro and Blier, 1999). However, this may not be the case because lowering serotonin availability does not always increase responses to threat. For example, depleting serotonin precursor does not increase, but decreases the recognition of fearful faces (Harmer et al, 2003b). Alternatively, results from two animal models suggest that the anxiogenic effect of SSRIs could be mediated by activation of $5 \mathrm{HT}_{2 c}$ (Bagdy et al, 2001). An indirect mechanism could also be responsible. Acute citalopram can increase cortisol levels (Attenburrow et al, 2001). Given that cortisol can facilitate fear in the amygdala and the BNST (Schulkin et al, 2005), it is possible that increased fear in the present study was not due to a direct effect of serotonin, but to the facilitation of amygdalaand/or BNST-mediated fear by cortisol.

To summarize, acute citalopram increased the expression of fear-related responses to different types of stimuli. This effect is consistent with clinical reports of symptom exacerbation following treatment initiation (Gorman et al, 1987; Ramos et al, 1993) and with evidence of facilitation of processing threat cues in experimental models following acute SSRIs (Burghardt et al, 2004; Harmer et al, 2003b). Fear-potentiated startle is therefore a useful tool to examine the effects of SSRI agent. Because the efficacy of SSRIs develops over time, future studies should examine whether the expression of fear-potentiated startle is reduced by chronic SSRIs in both humans and animals. Such an integrative approach will provide insight into the mechanisms underlying the therapeutic effects of SSRIs.

\section{ACKNOWLEDGEMENTS}

This research was supported by the Intramural Research Program of the National Institutes of Mental Health.

\section{REFERENCES}

Attenburrow MJ, Mitter PR, Whale R, Terao T, Cowen PJ (2001). Low-dose citalopram as a 5-HT neuroendocrine probe. Psychopharmacology 155: 323-326.

Bagdy G, Graf M, Anheuer ZE, Modos EA, Kantor S (2001). Anxiety-like effects induced by acute fluoxetine, sertraline or $\mathrm{m}$-CPP treatment are reversed by pretreatment with the 5-HT2C receptor antagonist SB-242084 but not the 5-HT1A receptor antagonist WAY-100635. Int $J$ Neuropsychopharmacol 4: 399-408.

Burghardt NS, Sullivan GM, McEwen BS, Gorman JM, LeDoux JE (2004). The selective serotonin reuptake inhibitor citalopram increases fear after acute treatment: but reduces fear with chronic treatment: a comparison with tianeptine. Biol Psychiatry 55: 1171-1178.

de Jong R, Groenink L, van der Gugten J, Olivier B (2002). The light-enhanced startle paradigm as a putative animal model of anxiety: effects of chlordiazepoxide, flesonoxan and fluvoxamine. Psychopharmacology 159: 176-180.

First MB, Spitzer RI, Williams JBW, Gibbon M (1995). Structured Clinical Interview for DSM-V (SCID). American Psychiatric Association: Washington, DC.

Funayama ES, Grillon C, Davis M, Phelps EA (2001). A double dissociation in the affective modulation of startle in humans effects of unilateral temporal lobectomy. J Cogn Neurosci 13: 721-729.

Gorman JM, Liebowitz MR, Fyer AJ, Goetz D, Campeas RB, Fyer MR et al (1987). An open trial of fluoxetine in the treatment of panic attacks. J Clin Psychopharmacol 7: 329-332.

Graeff FG, Viana MB, Mora PO (1997). Dual role of 5-HT in defense and anxiety. Neurosci Biobehav Rev 21: 791-799.

Griebel G, Moreau JL, Jenck F, Misslin R, Martin JR (1994). Acute and chronic treatment with 5-HT reuptake inhibitors differentially modulate emotional responses in anxiety models in rodents. Psychopharmacology 113: 463-470.

Grillon C, Ameli R (1998). Effects of threat of shock, shock electrode placement, and darkness on startle. Int J Psychophysiol 28: 223-231.

Grillon C, Ameli R, Woods SW, Merikangas K, Davis M (1991). Fear-potentiated startle in humans effects of anticipatory anxiety on the acoustic blink reflex. Psychophysiology 28: 588-595.

Grillon C, Baas JM (2003). A review of the modulation of startle by affective states and its application to psychiatry. Clin Neurophysiol 114: 1557-1579.

Grillon C, Baas JMP, Pine DS, Levine J, Lawley M, Ellis V (2005). The benzodiazepine alprazolam reduces contextual fear but not cued fear potentiated startle in humans. 60th Annual Meeting of the Society of Biological Psychiatry. Atlanta, GA.

Grillon C, Baas JMP, Pine DS, Lissek S, Lawley M, Ellis V et al (in press). The benzodiazepine alprazolam dissociates contextual fear from cued fear in humans as assessed by fear-potentiated startle. Biol Psychiatry (in press).

Grillon C, Baas JP, Lissek S, Smith K, Milstein J (2004). Anxious responses to predictable and unpredictable aversive events. Behav Neurosci 118: 916-924.

Harmer CJ, Bhagwagar Z, Cowen PJ, Goodwin GM (2002). Acute administration of citalopram facilitates memory consolidation in healthy volunteers. Psychopharmacology 163: 106-110.

Harmer CJ, Bhagwagar Z, Perrett DI, Vollm BA, Cowen PJ, Goodwin GM (2003a). Acute SSRI administration affects the processing of social cues in healthy volunteers. Neuropsychopharmacology 28: 148-152.

Harmer CJ, Rogers RD, Tunbridge E, Cowen PJ, Goodwin GM (2003b). Tryptophan depletion decreases the recognition of fear in female volunteers. Psychopharmacology 167: 411-417.

Hensman R, Gaimaraes FS, Wang M, Deakin JFW (1991). Effects of ritanserin on aversive classical conditioning in humans. Psychopharmacology 104: 220-224. 
Inoue T, Li XB, Abekawa T, Kitaichi Y, Izumi T, Nakagawa $\mathrm{S}$ et al (2004). Selective serotonin reuptake inhibitor reduces conditioned fear through its effect in the amygdala. Eur J Pharmacol 497: 311-316.

Inoue T, Tsuchiya K, Koyama T (1996). Serotonergic activation reduces defensive freezing in the conditioned fear paradigm. Pharmacol Biochem Behav 53: 825-831.

Kemp AH, Gray MA, Silberstein RB, Armstrong SM, Nathan PJ (2004). Augmentation of serotonin enhances pleasant and suppresses unpleasant cortical electrophysiological responses to visual emotional stimuli in humans. Neuroimage 22: 10841096.

Lino-de-Oliveira C, Sales AJ, Del Bel EA, Silveira MC, Guimaraes FS (2001). Effects of acute and chronic fluoxetine treatments on restraint stress-induced Fos expression. Brain Res Bull 55: 747-754.

Lissek S, Dvir S, Baas JMP, Mc Dowell DJ, Pine DS, Shaywitz E et al (2005a). Pathological anxiety is associated with sustained anxiety to an unpredictably stressful context but not with phasic fear reactions to an explicit threat-cue. 60th Annual Meeting of the Society of Biological Psychiatry. Atlanta, GA.

Lissek S, Powers AS, McClure EB, Phelps EA, Woldehawariat G, Grillon C et al (2005b). Classical fear conditioning in the anxiety disorders a meta-analysis. Behav Res Ther 43: 1391-1424.

Morris JS, Ohman A, Dolan RJ (1998). Conscious and unconscious emotional learning in the human amygdala. Nature 393: 467-470.

Noble S, Benfield P (1997). Citalopram: a review of its pharmacology, clinical efficacy and tolerability in the treatment of depression. CNS Drugs 8: 410-431.
Phelix CF, Liposits Z, Paull WK (1992). Serotonin-CRF interaction in the bed nucleus of the stria terminalis: a light microscopic double-label immunocytochemical analysis. Brain Res Bull 28: 943-948.

Phelps EA, O’Connor KJ, Gatenby JC, Anderson AK, Grillon C, Davis $M$ et al (1998). Activation of the human amygdala by a cognitive representation of fear: an FMRI study. Soc Neurosci Abstr 24: 1524.

Piñeyro G, Blier P (1999). Autoregulation of serotonin neurons: role in antidepressant drug action. Pharmacol Rev 51: 533-591.

Pollock BG (2001). Citalopram: a comprehensive review. Expert Opin Pharmacother 2: 681-698.

Ramos TT, Gentil V, Gorenstein C (1993). Clomipramine and initial worsening in panic disorder: beyond the "jitterness syndrome'. J Psychopharmacol 7: 265-269.

Sanchez C, Meier E (1997). Behavioral profiles of SSRIs in animal models of depression, anxiety and aggression. Are they all alike? Psychopharmacology 129: 197-205.

Schulkin J, Morgan MA, Rosen JB (2005). A neuroendocrine mechanism for sustaining fear. Trends Neurosci 28: 629-635.

Silva M, Hetem LA, Guimaraes FS, Graeff FG (2001). Opposite effects of nefazodone in two human models of anxiety. Psychopharmacology (Berlin) 156: 454-460.

Spielberger CD (1983). Manual for the State-Trait Anxiety Inventory. Consulting Psychologist Press: Palo Alto, CA.

Vertes RP, Fortin WJ, Crane AM (1999). Projections of the median raphe nucleus in the rat. J Comp Neurol 407: 555-582.

Walker DL, Toufexis DJ, Davis M (2003). Role of the bed nucleus of the stria terminalis versus the amygdala in fear, stress, and anxiety. Eur J Pharmacol 463: 199-216. 